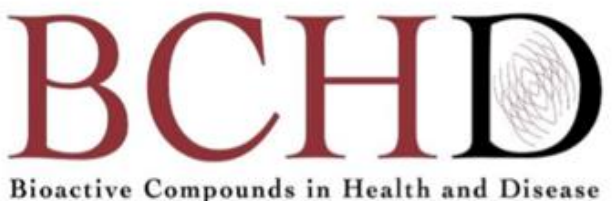

\title{
The emerging potential of functional foods in viral disease prevention
}

\section{Danik Martirosyan}

Functional Food Center, Functional Food Institute, Dallas, TX, USA

Submission Date: June 27 $7^{\text {th }}, 2020$; Acceptance Date: June $30^{\text {th }}, 2020$; Publication Date: June $30^{\text {th }}, 2020$

Please cite this article as: Martirosyan D.M, The Emerging Potential of Functional Foods in Viral Disease Prevention. Functional Foods in Health and Disease 2020; 6(10): 95-99. DOI: https://www.doi.org/10.31989/bchd.v3i6.726

\section{EDITORIAL}

Currently, functional foods are primarily used for the prevention and management of chronic diseases because the recommendation for long-term functional food consumption lines up with the longterm presence of chronic diseases and their symptoms in the body.

According to the Functional Food Center's (FFC) current definition, functional foods can be used for the prevention and management of chronic diseases and their symptoms. This existing, working definition provides a road map on how to create "ideal" functional food products, how to evaluate them, and how to use them for general health as well as for chronic diseases and their symptoms.

Though the Functional Food Center has suggested functional foods be studied and used for the management of chronic diseases, this editorial provides a viewpoint into using functional foods as a way to combat viral diseases as well. Through this editorial, we hope to propose an expansion of the currently established definition of functional foods to include this new use. The main reason we did not previously consider using functional foods to prevent or manage viral diseases was because from our previous knowledge, long-term consumption and usage of functional foods was necessary for the proper and effective action and effect. The impact of functional foods was understood as needing to build up certain micro- or macro- elements, vitamins, and other bioactive molecules in the body, to get a critical amount of bioactive compounds which will be effective but not toxic, as well as to start turning on some biologically known and understood processes on molecular, cellular, and organ levels. On the other hand, viral diseases are fast processes -- in contrast to chronic diseases -- and they usually develop within 
short periods, ranging from days to weeks. Therefore, it seemed the only logical and appropriate use of functional foods would be for treating chronic diseases and their symptoms.

However, recent developments with COVID-19 have changed our knowledge on the relationship between chronic diseases and viral diseases. Many people who were affected or have passed away from COVID-19 had one or more chronic diseases. Also, it became clear that those people who had several chronic diseases had weaker immune systems and were more susceptible to COVID-19 [1]]. Among that category many are elderly people whose immune system declined due to aging. So, in order to combat viral diseases, we can and should boost our immune system using different functional foods and bioactive compounds; in this way we will allow for and utilize functional foods in combating viral diseases and their spread. Here is some research supporting that potential functional food products could help enhance our immune system and help to combat viral diseases:

1. A very good example of bioactive compounds combating viruses is the efficacy of zinc in signaling antiviral activities by increasing the body's interferon response to help effectively fight viruses [2]. Zinc does not just possess direct antiviral properties but is also critical in propagating the innate and acquired antiviral responses [2]. Foods that are rich in zinc include, but are not limited to, green peas, spinach, asparagus, blackberries, pomegranate, and cantaloupe $[3,4]$.
2. In another study, authors from the UK show that in older people there was a greater specific antibody response to Pneumovax II vaccination in subjects who consumed 5 portions of fruits and vegetables per day than in subjects who consumed 2 portions fruits and vegetables per day [5]. This increased response was confined to subjects who had never previously received the Pneumovax II vaccination. Therefore, increased fruit and vegetable intake may improve the antibody response to Pneumovax II vaccination in older people. Although the specificity of the fruits and vegetables in the study is not clear, this finding links an achievable dietary goal with potentially enhanced protective immunity. This in and of itself is an encouraging endorsement of the 5-a-day message and could have important public health implications.

If scientists are to come up with new functional food recipes to combat viruses or to support the immune system, they will need to determine which bioactive compound(s) are effective for certain infections, determine exact and effective, but not toxic, amounts of bioactive compounds, and how long the functional food product should be consumed in order to enhance the immune system. The effect of functional foods, as well as bioactive compounds, should be measured by using specific biomarkers in clinical trials and by conducting future epidemiological investigations to prove the beneficial effect of functional foods on certain biomarkers for immunity. 
Historically, human nature seems to lead to selecting the solution that is easiest for getting the job done; in the case of viruses, therefore, most people prepare for viral diseases by getting a vaccine against the disease and hoping for protection. Of course, we are not implying that functional foods should take the place of vaccines, but functional foods should work alongside vaccines as a booster for the immune system in order to prevent viral and chronic diseases.

Here are some specific reasons why we should develop specific functional foods products for viral diseases:

1. Functional foods would be especially helpful in slowing the onset of diseases where the vaccine is not available (COVID-19) or where the previously created vaccine is not necessarily effective (certain strains of influenza). Even when the strain of a viral disease is identified, a vaccine can take years to develop and the benefits of functional foods that are consumed will be very helpful during the waiting period.

2. If a virus were to mutate to the point where a vaccine would no longer be effective, the consumption of functional foods would help to support the immune system when the vaccine cannot.

3. As we understand, the process of aging leads to an immune system decline, and that is the one of the major reasons why one of the most vulnerable populations infected by COVID-19 is elderly people. There is also evidence that immunization of elderly people is not always successful due to the weak immune system of that population. Using certain functional foods to boost the immune system would be an alternative option to fight with chronic diseases as well as viral diseases.

4. Since functional foods are considered as a regular or fortified food enriched with bioactive compounds, it's less likely that they have side effects, even though the research and development of that functional food, as well as the interaction between functional foods and medicine, should still be thoroughly investigated.

The use of functional foods to combat viral diseases would especially benefit the elderly, which has become a growing sector of the world population. The total population of the world is currently experiencing an increase in average age as well as a global aging phenomenon [6], where more and more people are living longer lives. Together, this means that it's more important than ever to a find healthy, safe, and productive ways of accommodating an aging population. A common side effect of aging is immune system decline, which is also why the elderly and immunocompromised are the most susceptible to the current COVID-19 epidemic. Vaccines also often end up not being effective for the elderly for these same reasons, as the standard doses in vaccines are not strong enough for the weaker immune systems of the elderly [7]. Functional foods would be highly effective here to help strengthen the immune system in order to fight COVID-19 and other viral diseases. Here is one example: Zinc deficiency, which leads to viral disease susceptibility, is also a common 
symptom of old age and as we stated earlier, zinccontaining foods served very well to improve the efficacy of the immune system in the face of several viral diseases [2]. Other examples of functional foods that are effective as an immune booster are fish oil (containing Omega-3 fatty acids), magnesiumcontaining foods (bananas, green beans, etc.), and Vitamin A-containing foods (apricots, broccoli, etc.) [8]. Functional foods wouldn't be a one-shot wonder that suddenly jump-starts one's immune system and makes them automatically immune to COVID-19 or any other viral disease, but rather foods that are to be consumed over time as a form of risk management to help with combating both viral and chronic diseases. Beginning the regular consumption of functional foods now will prepare the growing elderly population should another pandemic occur in the future. Functional foods provide long-term immune strength, are easy to obtain and consume, and benefit a growing sector of the world population, and therefore they should be considered as a priority when looking at ways to prevent viral diseases. We can conclude that functional foods could be effective for the prevention of viral diseases including COVID19.

In this editorial, we discussed the established definition of functional foods and their current use and then expanded on the established definition by exploring a new use for functional foods--using functional foods to fight viral diseases while alleviating and preventing chronic diseases. We found strong evidence corresponding to how functional foods and bioactive compounds could play a keystone role in strengthening the immune system to better fight viral diseases alongside chronic diseases. Using functional foods to prevent viral diseases would be a major public health breakthrough as it would be valuable in preventing countless deaths in the event of another pandemic, especially in the case where the strengths of vaccines are limited. Because of the magnitude of its effects, using functional foods against viral diseases should be considered as a major component of the definition of functional foods as well.

Accordingly we suggest making certain changes in FFC's definition of Functional foods, which first time we provided at 6 th International Conference of FFC "Functional Foods for Chronic Diseases: Diabetes and Related Diseases", December 4-5, 2009, Texas Woman's University, Denton, TX, USA and article on new definition of Functional foods was published in conference book [9]. Here is FFC's new version of functional food definition:

Functional Food Center defines "functional foods" as "Natural or processed foods that contain biologicallyactive compounds; which, in defined, effective, nontoxic amounts, provide a clinically proven and documented health benefit utilizing specific biomarkers, for improving general health, for the prevention, management and treatment of chronic and viral disease or its symptoms.

Acknowledgement: Thank you to FFC's interns Collete Chang (student from Johns Hopkins University) for helping to collect some information from literature and editing, and Jack Liufu (student from Cornell University) for editing and proofreading this editorial. 


\section{List of Abbreviations: FFC: Functional Food Center}

Competing Interests: The authors declare no competing interests.

Authors Contributions: Danik Martirosyan wrote the Editorial.

\section{REFERENCES:}

1. Butler MJ, Ruth M .Barrientos R.M. The impact of nutrition on COVID-19 susceptibility and long-term consequences. Brain, Behavior, and Immunity; Volume 87, July 2020, Pages 53-54

2. Read SA, Obeid S, Ahlenstiel C, Ahlenstiel G. The Role of Zinc in Antiviral Immunity. Advances in Nutrition. 10(4):696-710.

DOI: http://doi:10.1093/advances/nmz013.

3. Whitbread D. Top 10 Vegetables Highest in Zinc. myfooddata.

https://www.myfooddata.com/articles/high-zinc-

vegetables.php. Published April 9, 2020. Accessed June 17, 2020.

4. Whitbread D. Top 10 Fruits Highest in Zinc. myfooddata. https://www.myfooddata.com/articles/high-zincfruits.php. Published April 9, 2020. Accessed June 17, 2020.

5. Effect of fruit and vegetable consumption on immune function in older people: a randomized controlled trial. Gibson A, Edgar J.D, Neville C.E, Gilchrist S, McKinley M.C, Patterson C.C, Young I.S, Woodside J.V. The American Journal of Clinical Nutrition, Volume 96, Issue 6, December 2012, Pages 1429-1436,

DOI: http://doi.org/10.3945/ajcn.112.039057

6. Alpopi C, Nica E, Oancea MDN, Balu PE. Aspects of Sustainable Development in the Perspective of the Population Aging Phenomenon. Calitatea, Volume 20, Issue S2, March 2019, Pages 21-26. https://search.proquest.com/docview/2198414650?acco untid=10267. Accessed June 29, 2020.
7. Bacon CJ, Gamble GD, Reid IR. High-dose oral vitamin D3 supplementation in the elderly. Osteoporosis International. 2009; 20:1407-1415.

DOI: http://doi:10.1007/s00198-008-0814-9.

8. Vlachos A., Vassiliadis S. The immunological impact of orthomolecular medicine using bioactive compounds as key factors in endometriosis. Bioactive Compounds in Health and Disease 2019; 2(1): 1-10.

DOI: https://doi.org/10.31989/bchd.v2i1.555

9. Martirosyan D, Prasad C: Functional Foods for Chronic Diseases: Diabetes and Related Diseases. In The 6th International Conference proceeding 